\title{
Technical considerations in quantitative blood oxygenation measurement using photoacoustic microscopy in vivo
}

Konstantin Maslov, Mathangi Sivaramakrishnan, Hao F. Zhang, George Stoica, Lihong V. Wang

Konstantin Maslov, Mathangi Sivaramakrishnan, Hao F. Zhang, George Stoica, Lihong V. Wang, "Technical considerations in quantitative blood oxygenation measurement using photoacoustic microscopy in vivo," Proc. SPIE 6086, Photons Plus Ultrasound: Imaging and Sensing 2006: The Seventh Conference on Biomedical Thermoacoustics, Optoacoustics, and Acousto-optics, 60860R (6 March 2006); doi: 10.1117/12.646265 


\title{
Technical Considerations in Quantitative Blood Oxygenation Measurement Using Photoacoustic Microscopy in Vivo
}

\author{
Konstantin Maslov*, Mathangi Sivaramakrishnan, Hao F. Zhang, George Stoica ${ }^{\text {a }}$, Lihong V. Wang \\ Optical Imaging Laboratory, Department of Biomedical Engineering \\ Texas A\&M University, 3120 TAMU, College Station, Texas, USA 77843-3120 \\ ${ }^{\mathrm{a}}$ Department of Veterinary Pathobiology \\ Texas A\&M University, 5547 TAMU, College Station, Texas 77843-5547
}

\begin{abstract}
Using peak amplitude spectral PA measurements in the range of $570-600 \mathrm{~nm}$, we found it possible to quantify blood oxygenation levels. Visible light illumination minimizes the inversion error of the PA measurements. Owing to high blood absorption in this optical regime, there is also an improved signal-to-noise ratio and less influence from optical scattering. To arrive at correct, and vessel size independent, $\mathrm{SO}_{2}$ measurements, the central frequency of the ultrasonic transducer must be high enough to satisfy the relation $\mu_{a} \Lambda<1$, that is above $25 \mathrm{MHz}$ for a chosen optical wavelength region, although lower frequency transducers may produce correct results after correction of the optical absorption spectra. However, additional efforts are needed to achieve accurate $\mathrm{SO}_{2}$ values for in vivo measurements.
\end{abstract}

Keywords: Photoacoustic microscopy, Functional imaging, Spectroscopic imaging, Blood oxygenation

\section{INTRODUCTION}

The quantification of localized chromophore concentrations in biological tissues from signature optical absorption spectra has a wide range of applications in the biomedical field. Hemoglobin is an important chromophore that exists in two distinct forms, and the concentration ratio of the two forms defines the oxygen carrying capacity of blood. Research has shown that oxygenation level affects the healing of burns ${ }^{1}$ and wounds ${ }^{2}$ and determines the effectiveness of chemo- and radio-therapy ${ }^{3}$ for cancer. Hence, localized noninvasive oxygenation measurements in skin are critical in dermatology, cancer research and plastic surgery. Currently, pulse oximetry, based on near infra-red spectroscopy (NIRS) is widely used in clinical settings although this technique yields single point measurements of arterial blood oxygenation only and, therefore, lacks spatial resolution ${ }^{4}$.

Photoacoustic (PA) imaging is a novel modality that employs laser induced acoustic waves based on the principle of thermal expansion following optical energy absorption by the sample. Since the pressure generated is directly proportional to the local light absorption in the tissue, spectral PA measurements provide optical spectroscopic information ${ }^{5}$.

Most research efforts reported so far on extracting blood oxygenation levels from PA measurements in vitro utilize the exponential rise in PA signals. This approach, however, is not feasible for micro-vascular imaging in vivo, where peak amplitude value of PA signal from different structures is frequently the only parameter that can be separated in time. Here we report on some experimental considerations for blood oxygenation $\left(\mathrm{SO}_{2}\right)$ level measurements in subcutaneous vessels in vivo from peak amplitudes of spectral PA measurements obtained using a photo-acoustic system, and also analyze the factors that affect the accuracy of these measurements.

Photons Plus Ultrasound: Imaging and Sensing 2006: The 7th Conference on Biomedical

Thermoacoustics, Optoacoustics, and Acousto-optics, edited by A. A. Oraevsky, et. al

Proc. of SPIE Vol. 6086, 60860R (2006) • 1605-7422/06/\$15 • doi: 10.1117/12.646265 


\section{MATERIALS AND METHODS}

\subsection{Experimental equipment}

Figure 1(a,b) show the experimental setup of our photoacoustic microscopy system. A Q-switched pulsed Nd:YAG laser (Brilliant B, BigSky) acts as the pumping source for a tunable dye laser (ND6000, Continuum), which delivers, after attenuation, $3 \mathrm{~mJ}$ light pulses to a $0.62-\mathrm{mm}$ diameter optical fiber. The laser pulse width is $6.5 \mathrm{~ns}$, and the pulse repetition rate is $10 \mathrm{~Hz}$. The fiber output is positioned coaxially with a focuses ultrasonic transducer (Panametrics) on a three-dimensional precision mechanical scanner, as shown in Fig. 1b. The transducer has a center frequency of $50 \mathrm{MHz}$ with a nominal bandwidth of $70 \%$; it is attached to an in-house-constructed concave lens (aperture diameter $\mathrm{D}=5.5 \mathrm{~mm}$ and free working distance $\mathrm{L}$ $=5.6 \mathrm{~mm}$ ). The laser light from the fiber is expanded by a conical lens and then focused through an optical condenser with an NA of 1.1. The optical focal region overlaps with the focal spot of the ultrasonic transducer, thus forming a confocal optical dark-field illumination and ultrasonic detection configuration. Side illumination renders the assumption of omni-directional diffuse illumination of the imaging volume plausible.
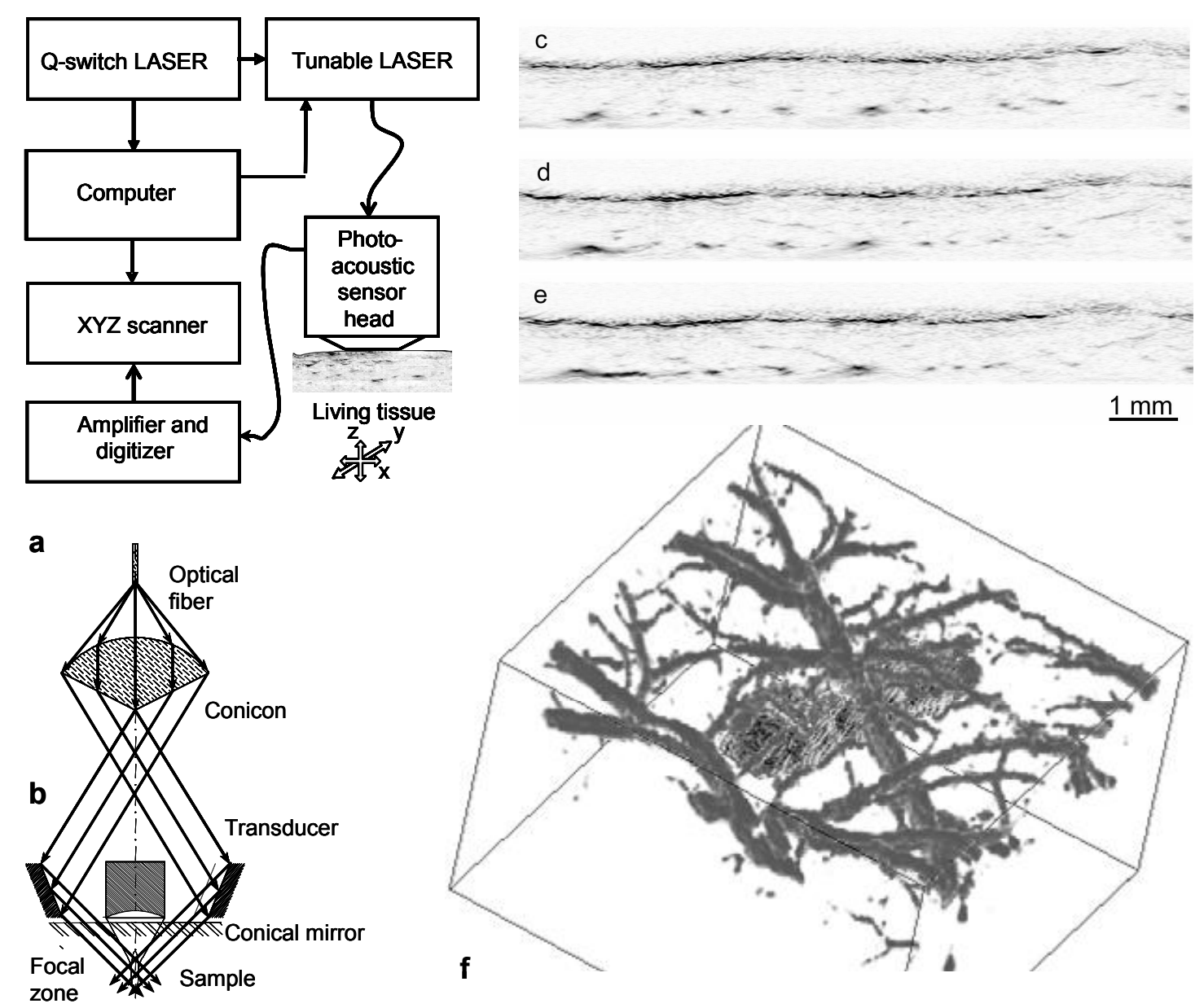

Figure 1 Photoacoustic microscopy system; (a) schematic of the imaging system, (b) diagram of the photoacoustic probe, (c),(d),(e) consecutive cross-sectional photoacoustic images (B-scans) of rat vasculature, (f) 3D image of rat vasculature near melanoma tumor reconstructed from series of B-scan photoacoustic images. 
Photoacoustic signals received by the ultrasonic transducer are amplified by a low-noise amplifier (ZFL500LN, Mini-Circuits) and recorded by a digital oscilloscope. To avoid the influence of laser output power instability, part of the laser beam is diverted to a silicon photo-diode, which is used to normalize the photoacoustic signal. The transducer is immersed in water in a plastic container with an opening at the bottom that is sealed with a thin disposable polyethylene membrane. The sample (animal) is placed outside the container below the membrane, and the ultrasonic coupling is further secured by coupling gel. Water temperature in the container is stabilized at $38^{\circ} \mathrm{C}$.

Using a $50 \mathrm{MHz}$ transducer (0.44 NA), the PAM system can image up to $3 \mathrm{~mm}$ deep with a lateral resolution of 40-120 $\mu \mathrm{m}$ in vivo depending on the imaging depth, and high axial resolution of 15 to $30 \mu \mathrm{m}, 7$. With other transducers used in this study $(25 \mathrm{MHz}, 0.26 \mathrm{NA}$ and $10 \mathrm{MHz}, 0.26 \mathrm{NA})$, the resolution degrades with the transducer central frequency and numerical aperture. Time history of photoacoustic signal recorded at each position along tissue surface forms so-called A-line, peaks of which are related to boundaries of the optically absorbing structures, peak amplitudes to absorption coefficients and arrival times to the distance to absorber along ultrasonic transducer axis. A gray-scale plot of the envelopes of time histories of the received photoacoustic signals versus the one-dimensional transducer position along scanning axis parallel to the skin surface forms B-scan image several of which are shown in Fig. 1 (c,d,e). Dark spots in Fig. 1(c-e) are image of blood vessels crossing B-scan planes. Combination of consecutive B-scans forms full in vivo 3D image of optically absorbing structures. A 3D image of melanoma tumor and surrounding peripheral vasculature in rat is shown in Fig 1(f). For image analysis, the various peaks along the A-lines of 3D image were identified and the corresponding spectral values were used for pixel-by-pixel calculation of $\mathrm{SO}_{2}$ by using published values of molar extinction coefficients for $\mathrm{HbO}_{2}$ and $\mathrm{HbR}^{8,9}$.

\subsection{Theoretical fundamentals}

Several parameters, such as sound absorption in biological media, vessel (modeled here by a cylindrical tube) diameter, ultrasonic transducer focal position, and illumination condition (which itself is a function of depth), tissue optical absorption, $\mu_{\mathrm{a}}$, and scattering, $\mu_{\mathrm{s}}$ can influence spectral PA measurements. Nevertheless, it can be reasonably stated that the magnitude of the PA signal from a blood vessel, $A_{k}\left(p_{\alpha}\right)$, which can be peak-topeak voltage or the peak amplitude of the transducer voltage, is a unique function of some tissue parameters, $p_{\alpha}$, including $\mathrm{HbR}$ and $\mathrm{HbO}_{2}$ concentrations $[\mathrm{HbR}]$ and $\left[\mathrm{HbO}_{2}\right]$. If the $\mathrm{PA}$ signal can be theoretically calculated from a known $p_{\alpha}$ at each wavelength, $\lambda_{k}$, the maximum likelihood estimate of the tissue parameters can be obtained by minimizing the difference between the experimentally measured, $A_{k}^{e}$, and the calculated PA signals, $A\left(\lambda_{k}\right)$, in the least squares sense. Mathematically, this can be shown as:

$$
\frac{\partial E}{\partial p_{\alpha}}=\frac{1}{n} \frac{\partial}{\partial p_{\alpha}} \sum_{k=1}^{n}\left(A_{k}^{e}-A\left(p_{\alpha}, \lambda_{k}\right)\right)^{2}=0 .
$$

The error function at convergence is non-zero due to the presence of true random errors (noise), which can be nullified by repeating the measurements, and the presence of systematic or modeling errors, which are caused by the influence of neighboring absorbers, sample inhomogeneity, process nonlinearity, errors in the theoretical model, and so on. Repetitive measurements do not decrease systematic errors, and in principle, systematic errors must be estimated independently which seems to be extremely complicated and virtually impossible in biological systems where one cannot, for example, replace a structure of interest under investigation with a known target and perform a complete system calibration. However, some estimate of unbiased systematic error can be made using the error at convergence as an estimator, and for lack of better knowledge, systematic errors can be assumed to be normally distributed and independent of optical 
wavelength. In vitro data shows almost zero bias; however, in vivo measurements produce significant bias, which will be discussed later in detail.

Taylor series linear expansion can be used to express relative changes in measured PA signals $\Delta A_{k}$ with changes in tissue parameters $\Delta p_{\alpha}$ :

$$
\Delta A_{k}=p_{j} \frac{\partial A_{k}}{\partial p_{j}} \frac{\Delta p_{\alpha}}{p_{j}}=S_{k \alpha} \frac{\Delta p_{\alpha}}{p_{j}},
$$

where $S_{J k}=p_{J} \frac{\partial A_{k}}{\partial p_{J}}$ (with no summation over capital letter indexes) are the so-called sensitivities of the PA signal at wavelength $k$ to parameter $J$. Eq. (2) constitutes a linear regression problem for errors in the inverted parameters with well known solution:

$$
\Delta p_{\alpha}=\left(S_{\beta k} S_{k \alpha}\right)^{-1} S_{\beta l} \Delta A_{l}^{e} .
$$

Assuming that measurement error is a wavelength independent additive error with variance $\sigma_{A}^{2}=\hat{E}$, where $\hat{E}$ is the residual value of $E$ at convergence, (3) can be used to estimate covariance of inverted parameters:

$$
\Delta p_{\alpha}\left(S_{\beta k} S_{k \alpha}\right) \Delta p_{\beta} \approx \hat{E} \text {. }
$$

Consequently, the variance bounds for a single parameter $\Delta p_{Z}$ are proportional to residual error $\sqrt{\hat{E}}$ multiplied by the error propagation factor $(\mathrm{EPF}){ }^{10}$ for $\Delta p_{Z}$ :

$$
\Delta p_{Z} \approx \pm\left[C_{Z Z}-C_{Z \xi} C_{\xi \zeta}^{-1} C_{\zeta Z}\right]^{-1 / 2} \sqrt{\hat{E}}
$$

where $C_{\beta \alpha}=S_{\beta k} S_{k \alpha}, \xi, \zeta=1,2, \ldots, Z-1, Z+1, Z+2, \ldots, \eta$ and $\eta$ is the number of independent parameters.

Error in inverted parameters (5) depends on a combination of the sensitivities $S_{j k}$ which are defined by the theoretical model used to calculate the PA signal. The PA signal is calculated from the published spectra of molar extinction for $\mathrm{HbR}$ and $\mathrm{HbO}_{2}$, that is, $\varepsilon_{a, H b R}(\lambda)$ and $\varepsilon_{a, H b O_{2}}(\lambda)$, respectively, using a linear regression technique ${ }^{11}$ that assumes that the PA signal is linearly proportional to the optical absorption of hemoglobin and the optical fluence at the blood vessel surface:

$$
\left[\begin{array}{c}
A_{\lambda_{1}} \\
A_{\lambda_{2}} \\
\vdots \\
A_{\lambda_{n}}
\end{array}\right]=K\left[\begin{array}{ccc}
\varepsilon_{a, H b O_{2}}\left(\lambda_{1}\right) & & \varepsilon_{a, H b R}\left(\lambda_{1}\right) \\
\varepsilon_{a, H b O_{2}}\left(\lambda_{2}\right) & & \varepsilon_{a, H b R}\left(\lambda_{2}\right) \\
& \vdots & \\
\varepsilon_{a, H b O_{2}}\left(\lambda_{n}\right) & & \varepsilon_{a, H b R}\left(\lambda_{n}\right)
\end{array}\right] \cdot\left[\begin{array}{c}
{\left[H b O_{2}\right]} \\
{[H b R]}
\end{array}\right],
$$

where, $\mathrm{K}$ is the unknown coefficient of proportionality between the incident light intensity and the PA signal magnitude, believed to be wavelength independent. Equation (6) can be inverted for hemoglobin concentrations $[\mathrm{HbR}]$ and $\left[\mathrm{HbO}_{2}\right]$, and $\mathrm{SO}_{2}=\left[\mathrm{HbO}_{2}\right] /\left(\left[\mathrm{HbO}_{2}\right]+[\mathrm{HbR}]\right)$ can be calculated by matrix inversion as a function of the known $\mathrm{Hb}$ spectra and the measured $\mathrm{PA}$ amplitudes. 


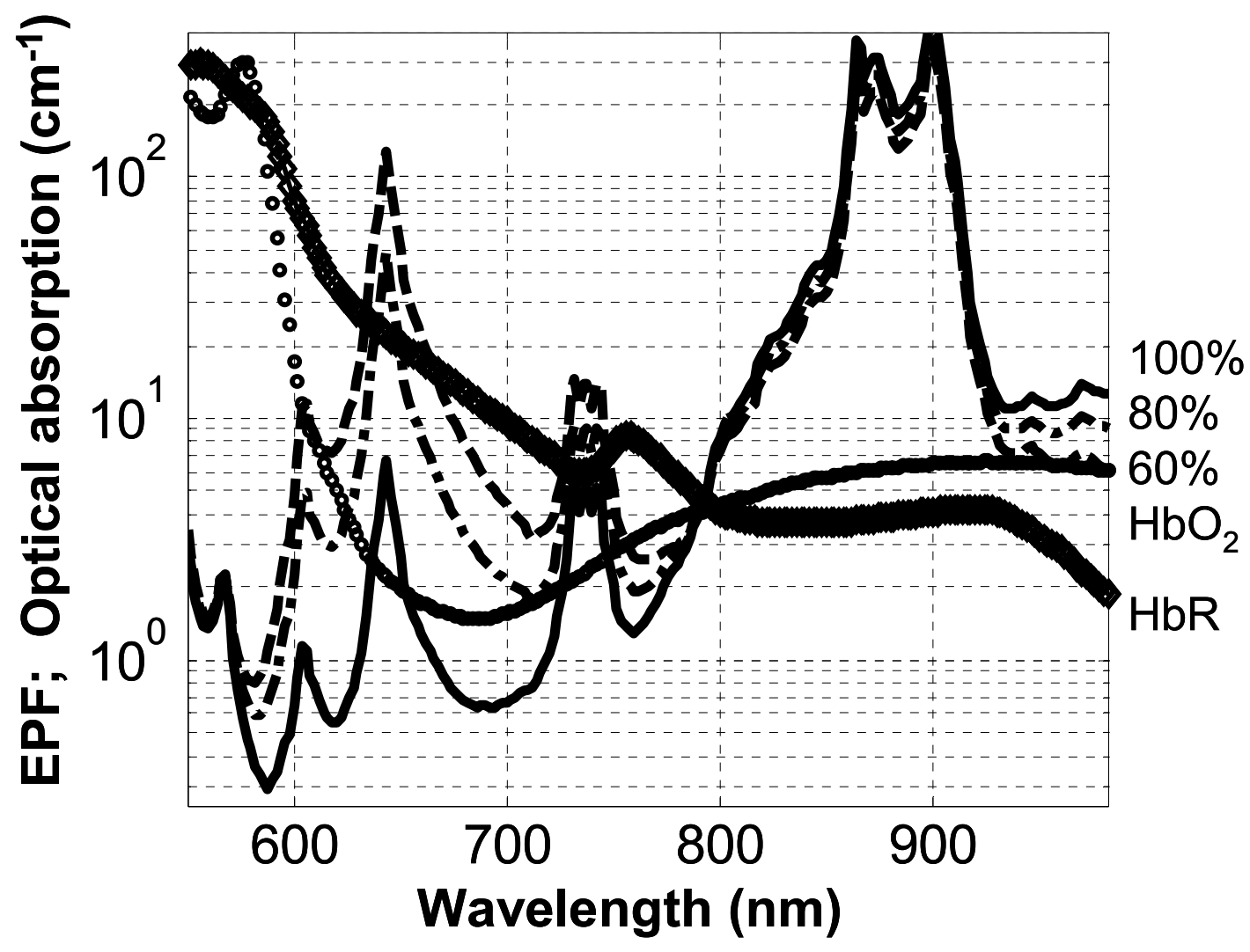

Figure 2 Error propagation factor (EPF) for the inversion of SO2 at different optical spectral bands. The solid lines show EPF at SO2 values ranging from $100 \%$ (thick solid line) to $60 \%$ in $20 \%$ steps; the $\mathrm{HbR}$ and $\mathrm{HbO} 2$ optical spectra are plotted for reference purposes.

Using (5) and (6), the EPF for $\mathrm{SO}_{2}$ can be estimated. The error propagation factors (EPF) for the $\mathrm{SO}_{2}$ inversion for four wavelengths $6 \mathrm{~nm}$ apart are plotted against the wavelength in Fig. 2 for $\mathrm{SO}_{2}$ values changing from $100 \%$ to $60 \%$ in steps of $20 \%$. The choice of narrow spectra; region alleviates the influence of other chromophores such as melanin, which have weak, but noticeable spectral dependence and also decrease the measurement time. Several distinct minima are noticeable around $575 \mathrm{~nm}, 785 \mathrm{~nm}$, and $1 \mu \mathrm{m}$. However, hemoglobin absorption at the NIR and IR wavelengths is almost two orders smaller than at $575 \mathrm{~nm}$, which increases the influence of noise and other absorbers, especially melanin and water. Moreover, at longer wavelengths, scattering is more pronounced owing to low blood absorption at these wavelengths, thus complicating the calculation of the PA signals from the blood vessels. Accordingly, the optical range of 578$596 \mathrm{~nm}$ has been chosen for in vivo $\mathrm{SO}_{2}$ measurements.

Equation (6) is valid only for small light absorption when $\mu_{a} a<<1$, where $a$ is the vessel radius and $\mu_{a}$ is the optical absorption in blood. However, in the chosen spectral range and transducer frequency band, it is not true for majority of detectable blood vessels. Correspondingly, prior to applying the method for in vivo measurements, the linearity of the PA signal amplitude dependence on $\mu_{a}$ for highly absorptive objects, $\mu_{a} a \geq 1$, must be experimentally proved. 


\section{RESULTS AND DISCUSSION}

\subsection{In vitro measurements}
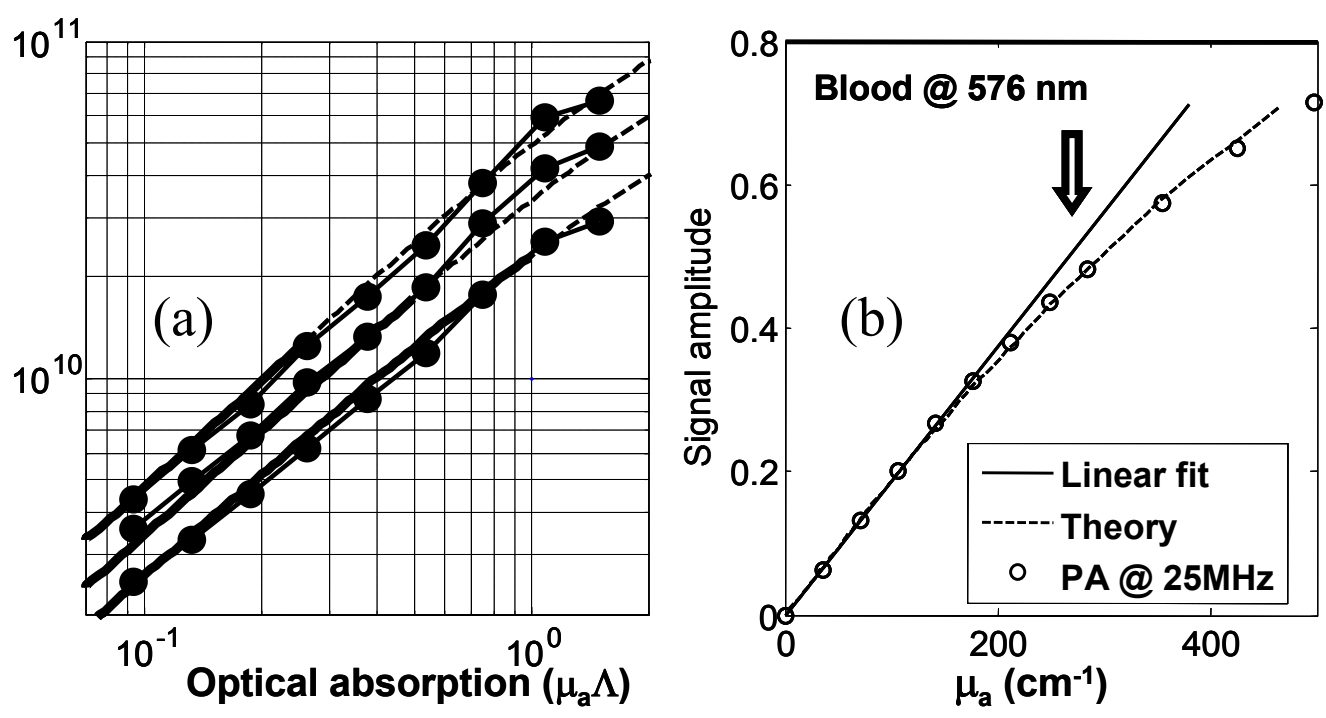

Figure 3 The peak magnitude of the photoacoustic pressure (a) at a large distance from an uniformly illuminated cylinder as a function of the normalized optical absorption for normalized cylinder radii a=0.83 $\Lambda$ (upper curve), $\mathrm{a}=0.625 \Lambda$, and $\mathrm{a}=0.42 \Lambda$ as compared to experimental data and (b) that from a $0.25 \mathrm{~mm}$ diameter cylinder tube versus the absorption coefficient for ink.

Fig. 3a shows the peak amplitude of experimentally measured PA signals from tubes of $0.25 \mathrm{~mm}, 0.5 \mathrm{~mm}$, and $1 \mathrm{~mm}$ diameter filled with ink (Lake Placid Blue, Private Reserve Ink) at various concentrations. Measurements were performed using a $5 \mathrm{MHz}$ transducer. This represents scaled model of blood vessel typically imaged by PAM; all problem length parameters - light absorption length, ultrasonic wavelength and cylinder radius are ten times bigger than in typical situation in vivo. Fig. 3b shows the peak amplitudes of the experimentally measured PA signals with 100 times averaging from a $0.25 \mathrm{~mm}$ diameter tube filled with ink samples of different concentrations covering the absorption range of whole blood in the visible spectrum. Measurements were acquired using a $50 \mathrm{MHz}$ central frequency transducer; however, due to frequency dependent ultrasonic absorption in the tube material, the measured central frequency was near 25 MHz. The experimental data coincide with a linear fit up to $\mu_{a} a \approx 2$. There is a good correspondence between the experimental and theoretical data calculated by convolving the experimentally measured transient response of the ultrasonic transducer with the time domain photoacoustic pressure for cylindrically symmetric problems ${ }^{12}$ with instant heat deposition for homogeneous cylinders uniformly illuminated by omni-directional scattered light given by:

$$
H\left(\hat{\mu}_{a}, \xi\right)=\frac{\mu_{a}}{\pi} \int_{0}^{\pi / 2} \int_{0}^{\pi} \exp \left(\mu_{a}\left(\xi \cos \theta-\sqrt{1-(\xi \sin \theta)^{2}}\right) / \sin \varphi\right) \cos \varphi d \theta d \varphi .
$$

Fig. 4 shows a plot of in vitro $\mathrm{SO}_{2}$ measurements performed on whole bovine blood using PAM against NIR spectrophotometric $\mathrm{SO}_{2}$ measurements at different oxygenation levels using $25 \mathrm{MHz}$ and $10 \mathrm{MHz}$ transducers. The PA measurements were performed on bovine blood samples of different oxygenation levels anti-coagulated with citrate dextrose solution. Two stock samples of fully oxygenated and deoxygenated blood were prepared by saturating the bovine blood with pure oxygen at $6^{\circ} \mathrm{C}$ and carbon dioxide at $36^{\circ} \mathrm{C}$, 
respectively. Four more blood samples at different oxygenation levels were prepared by mixing the two stock samples in preset volumetric ratios ${ }^{13}$. As a standard for comparison, spectrophotometric (GENESYS 20, Thermo Electron) measurements in the wavelength range of 700-1000 nm in steps of $4 \mathrm{~nm}$ were obtained on saponin-hemolyzed samples ${ }^{14}$. Single point measurements from the samples injected into tubes of $0.25 \mathrm{~mm}$ diameter were acquired using the PAM system at 12 wavelengths between 576 and $598 \mathrm{~nm}$ in steps of $2 \mathrm{~nm}$. The inversion technique previously described was used to quantify the $\mathrm{SO}_{2}$ level of each sample.

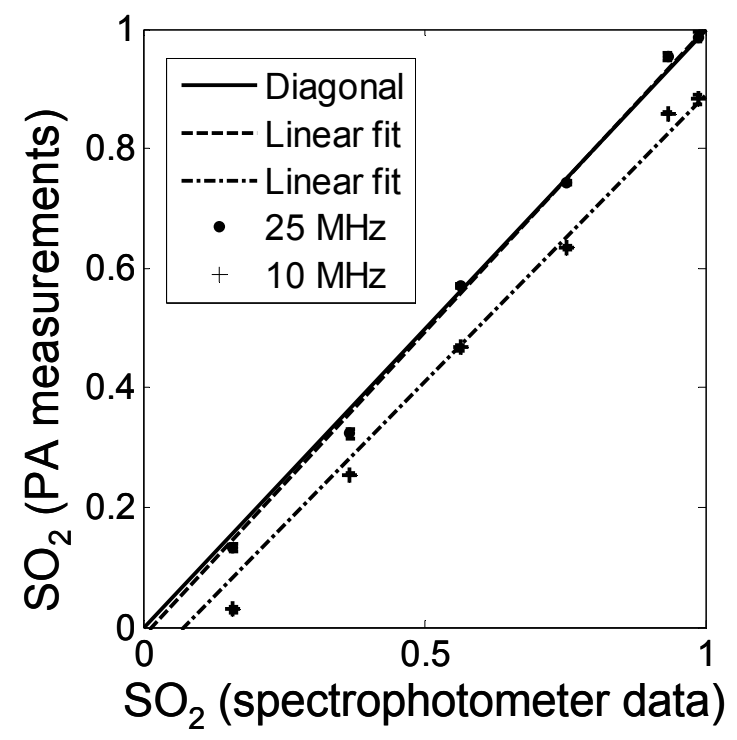

Figure 4 The inverted $\mathrm{SO}_{2}$ values from in vitro photoacoustic measurements vs. those from optical measurements using chemically hemolyzed blood

Measurements at $25 \mathrm{MHz}$ agreed with the optical measurements with a systematic error (accuracy) of $\sim 1 \%$, which may be fortuitous. Nevertheless, the variance of the PA measurements does not exceed 1\%, which indicates the high sensitivity of PAM in measuring $\mathrm{SO}_{2}$. Phantom and in vitro studies demonstrate high linearity in the PA peak amplitude measurements with respect to $\mu_{a}$ and high accuracy and sensitivity of $\mathrm{PAM}$ in quantitative measurements of $\mathrm{SO}_{2}$ within its realm of application. The effect of transducer focus on the $\mathrm{SO}_{2}$ measurements was found to be negligibly small. With a $25 \mathrm{MHz}$ transducer, the highest deviation of $\mathrm{SO}_{2}$ measured at $\pm 0.5 \mathrm{~mm}$ from the transducer focus from the $\mathrm{SO}_{2}$ value calculated at the focus does not exceed $2 \%$.

\subsection{In vivo measurements}

As the next progressive step, in vivo spectral PA measurements were conducted on Sprague Dawley rats ( 200 g, Charles River Breeding Laboratories). All experimental animal procedures were carried out in conformity with the guidelines of the United States National Institutes of Health ${ }^{15}$. The laboratory animal protocol for this work was approved by the ULAC of Texas A\&M University. Hair was removed from the region of interest prior to imaging using commercial hair remover lotion. Intramuscular injection of 87 $\mathrm{mg} / \mathrm{kg}$ Ketamine plus $13 \mathrm{mg} / \mathrm{kg}$ Xylasine was administered to anesthetize the rat, and the animal was kept motionless throughout the experiment with supplemental injections of a similar anesthetic mixture $(\sim 50$ $\mathrm{mg} / \mathrm{kg} /$ hour). The area to be imaged was fixed using an in-house made holder and water and ultrasound gel were used to provide ultrasound coupling. The body temperature of the animal was maintained throughout the experiment. Spectral images were obtained at the four wavelengths of $578 \mathrm{~nm}, 584 \mathrm{~nm}, 590 \mathrm{~nm}, 596 \mathrm{~nm}$. A pulse oximeter (Nonin Medical, Inc) was used to monitor the arterial blood saturation and heart rate of the animal. The animal recovered after the experiment without noticeable health problems. 
$578.5 \mathrm{~nm}$
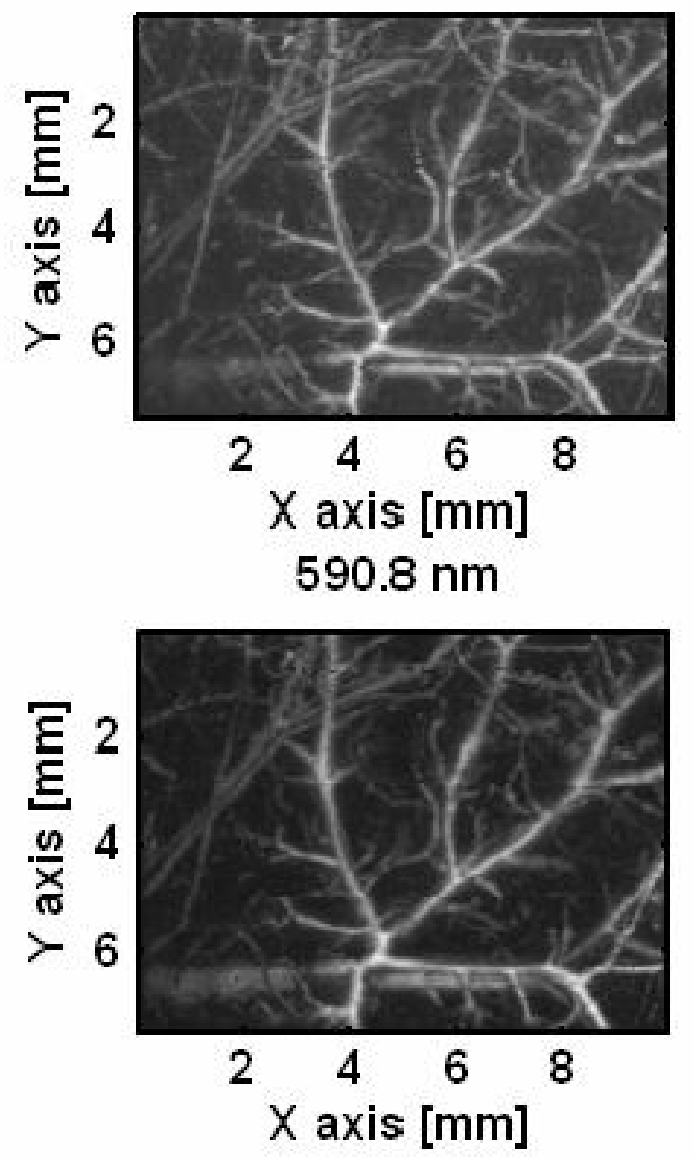

$584.7 \mathrm{~nm}$
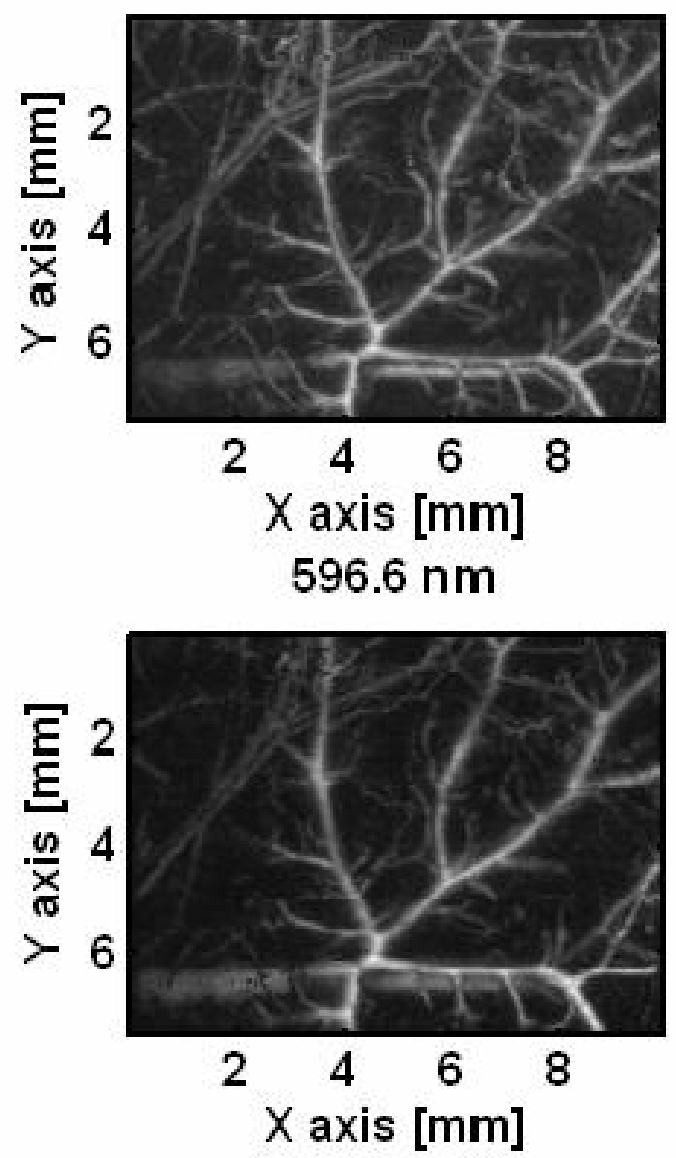

Figure $5 \mathrm{C}$-scan (maximum intensity projection) images of rat skin vasculature taken with a $50 \mathrm{MHz}$ transducer. Pixel brightness represents the PA intensity. Bar size is $1 \mathrm{~mm}$; each image was normalized on its maximum brightness value.

Figure 5 shows the photoacoustic maximum intensity projection images of the vascular distribution in the dorsal dermis (the upper lumbar area left of the vertebra) of Sprague Dawley rats taken with a $50 \mathrm{MHz}$ transducer at $578 \mathrm{~nm}, 584 \mathrm{~nm}, 590 \mathrm{~nm}$, and $596 \mathrm{~nm}$, respectively. Each image is normalized on its maximum brightness value. As one can see, different blood vessels have different relative contrast levels at short and long wavelengths, possibly due to different blood oxygenation levels in the veins and arteries. Data in Fig. 5 was used for $\mathrm{SO}_{2}$ measurements. The statistical distribution of the $\mathrm{SO}_{2}$ values over the area presented in Fig.5 is shown in Fig. 8. The $\mathrm{SO}_{2}$ data was weighted on PA amplitude at the isosbestic wavelength of $584 \mathrm{~nm}$ to discriminate erroneous data from areas between blood vessels. As one can see, not all measured $\mathrm{SO}_{2}$ lies within the physiologically possible range. Under normal conditions, the arterial blood oxygenation level is expected to be about $98 \%$, and the level in venous blood is about $89 \%{ }^{16}$. The measured $\mathrm{SO}_{2}$ values do not match these numbers, which indicates the influence of factors that may be specific to in vivo PA measurement. Most plausibly, the optical absorption caused by blood in the capillary network in skin tissue imparts its spectral signature on the fluence reaching the blood vessels of interest. These factors are defined by the tissue properties and must be measured independently. 


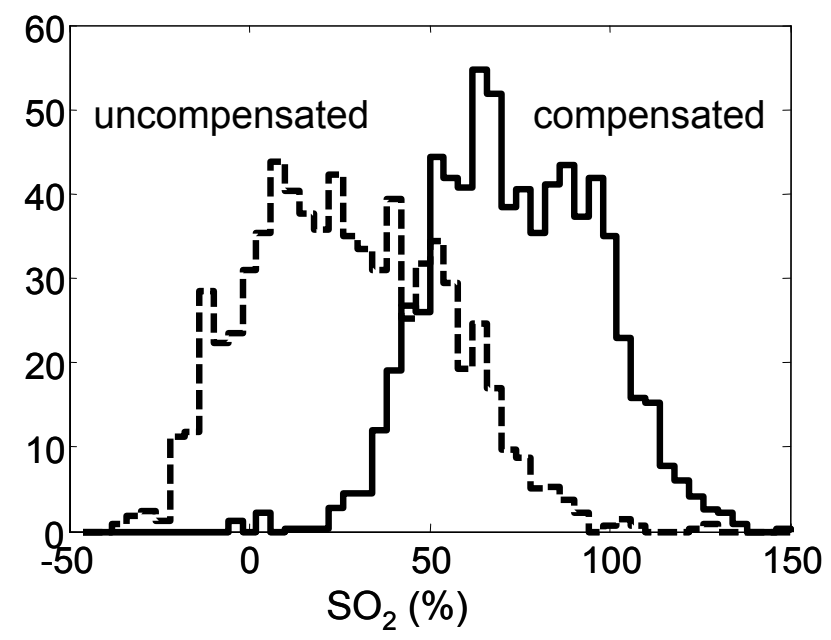

Figure 6 Weighted distribution of $\mathrm{SO}_{2}$ values for data from Fig.5.
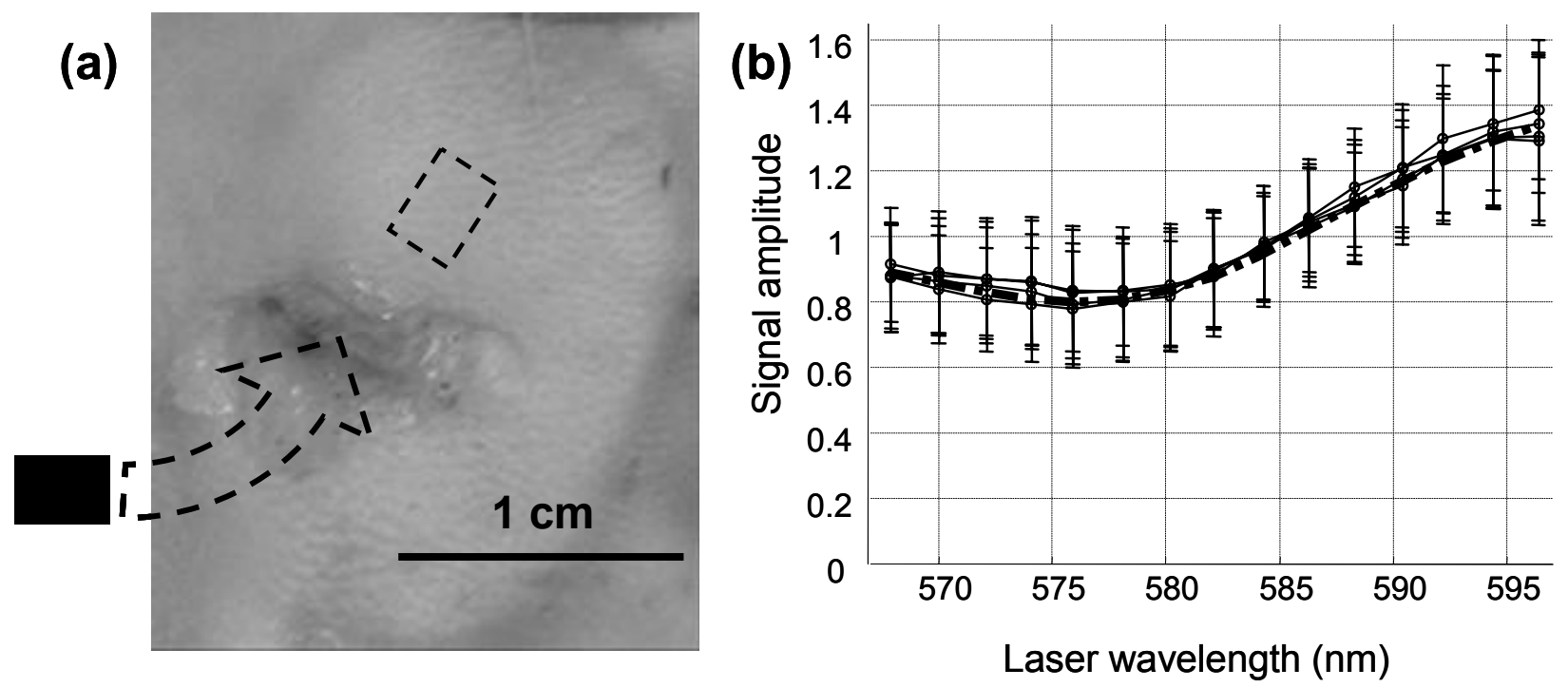

Figure 7. (a)Insertion of $50 \mu \mathrm{m}$ thick black polyethylene film under rat dermis and (b) the spectra of PA signals from the black insert taken in vivo.

To measure skin optical attenuation, black polyethylene film was inserted under the rat skin as shown in Fig. 7a. During the experiment, a Pulse Oximeter ( $8600 \mathrm{~V}$, Nonin Medical, MN) was used to monitor the heart rate and arterial blood oxygenation which was $98 \%$. PAM images of $2 \mathrm{~mm}$ X $2 \mathrm{~mm}$ area enclosing the black insert were acquired at 15 wavelengths. The spectra on Fig. $7 \mathrm{~b}$ are average spectra for different scanning lines normalized on their own mean magnitude. The dashed line corresponds to the best fit assuming exponential decay of optical fluence: $I=I_{o} \exp \left(-z \cdot[\mathrm{Hb}] \cdot \varepsilon\left(\lambda, S \mathrm{O}_{2}\right)\right)$, where the hemoglobin amount $z \cdot[\mathrm{Hb}]$ per unit area and average $\mathrm{SO}_{2}$ are unknown quantities, and $\varepsilon\left(\lambda, \mathrm{SO}_{2}\right)$ is molar extinction of hemoglobin at a given oxygenation level. As it can be seen, the PA signal amplitude varies significantly (up to $\pm 30 \%$ ) along the surface of the insert; however, PA optical wavelength dependence is preserved. The relative stability of the dermal spectral properties permits the following compensation technique. Owing to 
the large difference in the PA spectra of veins and arteries, one can easily identify them. Then, measured PA spectra can be corrected for fluence change $I=I_{o} \exp ^{\alpha}\left(-z \cdot[H b] \cdot \varepsilon\left(\lambda, S O_{2}\right)\right)$ where $\alpha$ is the ratio of $z \cdot[H b]$ in skin under investigation, to the same quantity in the case used to get the data in Fig. 7b. Since the $\mathrm{SO}_{2}$ change in arteries is very small, it is possible to find $\alpha$ by matching the inverted arterial $\mathrm{SO}_{2}$ with the pulse oximeter data and use it for the fluence compensation of other vessels. The resulting $\mathrm{SO}_{2}$ measurements yield physiologically plausible results as shown in Fig. 6.

\section{SUMMARY}

It is possible to quantify blood oxygenation levels from peak amplitude spectral PA measurements using optical wavelengths in the range of $570-600 \mathrm{~nm}$. Owing to high absorption of blood and negligible scattering, visible light illumination minimizes the inversion error of the PA measurements. To get vessel size independent $\mathrm{SO}_{2}$ measurements, high frequency transducers that satisfy the relation $\mu_{a} \Lambda<1$ are required. This necessitates the use of detecting transducer with center frequency above $25 \mathrm{MHz}$ for the chosen optical wavelength region. A technique to compensate for change in the optical fluence caused by diffuse optical attenuation in the skin has been proposed and tested.

\section{ACKNOWLEGEMENTS}

This project was sponsored by the National Institutes of Health grants R01 EB000712 and R01 NS46214. Wang's email address is LWang@tamu.edu. 


\section{REFERENCES}

1. B. Venkatesh, R. Meacher, M. J. Muller, T. J. Morgan, J. Fraser, “Monitoring tissue oxygenation during resuscitation of major burns,” J. Trauma, 50, 485-494(2001).

2. F. Gottrup, "Oxygen in Wound Healing and Infection," World J. Surg. 28, 312-315(2004).

3. M. Henke, C. Bechtold, F. Momm, W. Dorr, R. Guttenberger, "Blood haemoglobin level may affect radiosensitivity—preliminary results on acutely reacting normal tissues," Int. J. Radiation Oncology Biol. Phys. 48, 339-345(2000).

4. V. Kamat, "Pulse Oximetry," Indian J. Anaesth. 46, 261-268(2002).

5. X. Wang, Y. Pang, G. Ku, X. Xie, G. Stoica, and L. V. Wang, "Non-invasive laser-induced photoacoustic tomography for structural and functional imaging of the brain in vivo," Nat. Biotech. 21, 803-806 (2003).

6. K. Maslov, G. Stoica, and L. V. Wang, "In vivo dark-field reflection-mode photoacoustic microscopy,” Optics Letters, 30 (6) 625-627(2005).

7. K. Maslov, and L. V. Wang, "High-resolution photoacoustic vascular imaging in vivo using a large-aperture acoustic lens," Proc. SPIE 5697, 7-14 (2005).

8. S. L. Jacques and S. A. Prahl, “Absorption spectra for biological tissues,” (Oregon Medical Laser Center, Portland, Oreg., 2004), http://omlc.ogi.edu/spectra/haemoglobin/index.html. 9. W. G. Zijlstra, A. Buursma and O. W. van Assendelft, Visible and near infrared absorption spectra of human and animal haemoglobin, determination and application, (VSP, The Netherlands, 2000).

10. C. L. Yapura, V. K. Kinra, and K. Maslov, "Measurement Of Six Acoustical Properties Of A Three-Layered Medium Using Resonant Frequencies" J. Acoust. Soc. Am., 115, 57-65 (2004).

11. J. Laufer, C. Elwell, D. Delpy and P. Beard, "In vitro measurements of absolute blood oxygen saturation using pulsed near-infrared photoacoustic spectroscopy: accuracy and resolution,” Phys. Med. Biol. 50, 4409-4428 (2005).

12 G. J. Diebold, T. Sun, and M. I. Khan, “Photoacoustic Monopole Radiation in One, Two, and Three Dimensions," Physical Review Letters, 69 (9), 3384-3387, (1991) 13. P. Scheid, and M. Meyer, "Mixing technique for study of oxygen-haemoglobin equilibrium: a critical evaluation," J. Appl. Physiol. 45, 818-822 (1978).

14. M. U. Tsao, S. S. Sethna, C. H. Sloan, and L. J. Wyngarden, "Spectrophotometric determination of the oxygen saturation of whole blood," J. Biol. Chem. 217, 479-488 (1955).

15. National Institutes of Health, "Guide for the Care and Use of Laboratory Animals," NIH Pub. 86-23, (U.S. Government Printing Office, Washington DC, 1985).

16. H. Kobayashi and N. Takizawa, "Oxygen saturation and pH changes in cremaster microvessels of the rat," Am. J. Physiol. 270, H1453-H1461 (1996). 